\title{
Estimativa matemática do comportamento mecânico de compósitos de polipropileno com fibras de vidro
}

\author{
Eliana Contharteze Grigoletto, William de Melo Silva ${ }^{2}$ \\ Departamento de Bioprocessos e Biotecnologia, UNESP, Botucatu, SP \\ Rafael Pinatti ${ }^{3}$ \\ Instituto de Química, UNESP, Araraquara, SP
}

Resumo. Estimativas do comportamento mecânico de materiais compósitos viscoelásticos ${ }^{4}$ isotropicamente uniformes ${ }^{5}$ foram obtidas a partir de uma análise matemática dos dados $(\epsilon(t), \sigma(t))$, onde $\epsilon(t)$ é a porcentagem de deformação do corpo de prova no instante de tempo $t$, e $\sigma(t)$ é a tensão aplicada ao corpo de prova no instante de tempo $t$, obtidos a partir de $\mathcal{T}_{j}$ testes mecânicos, com $j=1, \ldots, k$, nas porcentagens de concentração $\gamma_{j}$ do material de reforço, respectivamente.

Palavras-chave. Compósitos de polipropileno, Fibras de vidro, Testes mecânicos.

\section{Introdução}

Partículas são aditivos importantes para alterar e melhorar as propriedades de uma matriz polimérica, como: condutividade, resistência ao desgaste, propriedades ópticas, entre outras $[2,3,5-8]$. Os compósitos estão no centro das atenções de muitas indústrias, laboratórios e pesquisas acadêmicas por resultarem em uma material com propriedades melhoradas.

Vários são os modelos matemáticos que procuram descrever a relação entre tensão e deformação, já que são dois conceitos importantes para a caracterização de um material compósito.

A lei básica de comportamento mecânico de materiais sólidos com características puramente elásticas, a lei de Hooke, diz que a tensão $\sigma(t)$ é diretamente proporcional a porcentagem de deformação $\epsilon(t)$, ou seja,

$$
\sigma(t)=\mathrm{E} \epsilon(t)
$$

\footnotetext{
${ }^{1}$ elianac@fca.unesp.br

${ }^{2}$ williamsilva@fca.unesp.br

${ }^{3}$ rafa_pinatti@hotmail.com

${ }^{4}$ Materiais mais leves e resistentes.

${ }^{5}$ Futuramente, pode-se estender esta pesquisa para o caso geral em que a tensão e a deformação forem campos tensoriais.
} 
onde E representa o módulo de Young ou módulo de elasticidade. A lei mecânica usada para modelar a relação entre tensão e deformação de fluidos é a lei de Newton, que diz que a tensão é proporcional a taxa de deformação, ou seja,

$$
\sigma(t)=\eta \frac{\mathrm{d}}{\mathrm{d} t} \epsilon(t)
$$

onde $\eta$ é a constante de viscosidade. A equação (2) é uma boa modelagem para fluidos Newtonianos: água, gases e a maioria dos líquidos. Não são bem representados por essa modelagem fluidos como: tintas, fluidos biológicos, entre outros.

$\mathrm{Na}$ natureza não existem materiais sólidos elásticos e fluidos que possam ser perfeitamente modelados pelas equações (1) e (2). Na prática, materiais compósitos viscoelásticos apresentam um comportamento intermediário entre o sólido elástico e o líquido viscoso, dessa forma, uma modelagem mais adequada pode ser obtida pela combinação das equações (1) e (2). Os modelos de [4]: Maxwell, Kelvin-Voigt, Zener, Maxwell modificado e Kelvin-Voigt modificado, modelam materiais combinando as equações (1) e (2).

Este trabalho está disposto da seguinte forma: na Seção 2 apresentamos uma curva típica de deformação $\times$ tensão de materiais compósitos que admitem características viscoelásticas quando submetidos à forças de deformação. Um ajuste de curvas para testes mecânicos está detalhado na Seção 3. Através do ajuste de curvas detalhado na Seção 3, estimamos valores de propriedades mecânicas de materiais não testados, tais como: tensão máxima de tração, limite de ruptura e os módulos de resiliência e Young na Seção 4. Encerramos este trabalho com as conclusões na Seção 5.

\section{Gráfico deformação $\times$ tensão}

O gráfico de curvas que representam a relação entre a deformação e a tensão são extremamente importantes na análise de propriedades mecânicas de uma material. Tais curvas podem sofrer substanciais variações, como podemos notar na Fig. 1.

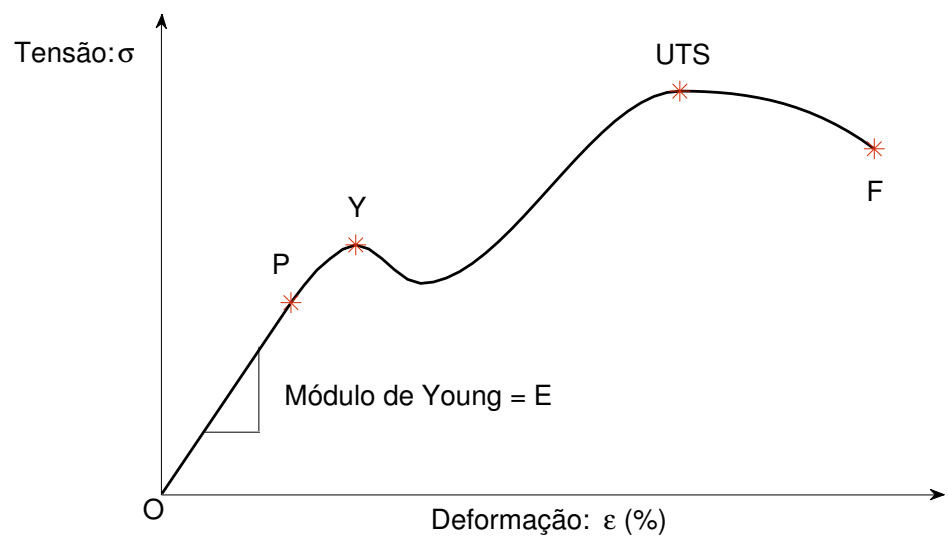

Figura 1: Típica curva de deformação $\times$ tensão de um compósito. 
Partindo da origem e seguindo pelo gráfico, alguns pontos estão indicados. O ponto $\mathrm{P}$ é conhecido como limite de proporcionalidade, até o ponto P a Lei de Hooke é teoricamente obedecida, e se a tensão aplicada ao material for removida, o mesmo retorna ao seu estado original. O ponto Y é conhecido como limite elástico, a partir dele, a tensão aplicada ao compósito resulta em uma deformação inelástica, ou seja, permanente. O ponto UTS indica a localização do valor de tensão máxima e o ponto $\mathrm{F}$ indica o ponto de fratura.

\section{Ajuste de curvas}

Seja $f(t)$ uma função real tal que para $i \in\{1,2, \ldots, m\}$, temos $\sigma_{i}=f\left(\epsilon_{i}\right)$. Dado um conjunto de pontos $\mathcal{S}=\left\{\left(\epsilon_{1}, \sigma_{1}\right),\left(\epsilon_{2}, \sigma_{2}\right), \ldots,\left(\epsilon_{m}, \sigma_{m}\right)\right\}$, onde temos valores arbitrários $\sigma_{1}, \sigma_{2}, \ldots, \sigma_{m}$ e $\epsilon_{i} \neq \epsilon_{j}$, para todo $i \neq j$, com $i, j \in\{1,2, \ldots, m\}$, vamos encontrar um polinômio ${ }^{6}$ de grau $n, p_{n} \in \mathbb{P}_{n}$, com $n+1 \leq m$, tal que

$$
p_{n}\left(\epsilon_{i}\right)=\sigma_{i}, \quad i=1,2, \ldots, m
$$

Se $p_{n} \in \mathbb{P}_{n}$, então

$$
p_{n}(x)=\sum_{j=0}^{n} a_{j} x^{j} .
$$

Substituindo a equação (3) na equação (4), temos

$$
p_{n}\left(\epsilon_{i}\right)=\sum_{j=0}^{n} a_{j}\left(\epsilon_{i}\right)^{j}=\sigma_{i},
$$

para $i=1,2, \ldots, m$. Assim, obtemos o seguinte sistema de $m$ equações em $n+1$ restrições, que pode ser escrito na forma matricial $\mathrm{AX}=b$, sendo:

$$
\mathrm{A}=\left[\begin{array}{ccccc}
1 & \epsilon_{1} & \left(\epsilon_{1}\right)^{2} & \cdots & \left(\epsilon_{1}\right)^{n} \\
1 & \epsilon_{2} & \left(\epsilon_{2}\right)^{2} & \cdots & \left(\epsilon_{2}\right)^{n} \\
\vdots & \vdots & \vdots & \ddots & \vdots \\
1 & \epsilon_{m} & \left(\epsilon_{m}\right)^{2} & \cdots & \left(\epsilon_{m}\right)^{n}
\end{array}\right]_{m \times(n+1)} \quad, \quad \mathrm{X}=\left[\begin{array}{c}
a_{0} \\
a_{1} \\
a_{2} \\
\vdots \\
a_{n}
\end{array}\right] \quad \text { e } b=\left[\begin{array}{c}
\sigma_{1} \\
\sigma_{2} \\
\vdots \\
\sigma_{m}
\end{array}\right]
$$

Em geral, o sistema $\mathrm{AX}=b$ não pode ser resolvido exatamente se $n+1<m$, e neste caso temos apenas que $\mathrm{AX} \cong b$. Agora, se $n+1=m$, como $\epsilon_{i} \neq \epsilon_{j}$, para todo $i \neq j$, podemos resolver exatamente o sistema e, neste caso, o vetor solução X é dado por

$$
\mathrm{X}=\mathrm{A}^{-1} b .
$$

\footnotetext{
${ }^{6} \mathbb{P}_{n}$ denota o conjunto dos polinômios de grau $n$ em $\mathbb{R}$ na base monomial $\mathcal{B}=\left\{1, x, x^{2}, \ldots, x^{n}\right\}$.
} 


\subsection{Testes mecânicos}

Sejam $\mathcal{T}_{j}$ testes mecânicos, com $j=1, \ldots, k$, nas porcentagens de concentração $\gamma_{j}$ do material de reforço, respectivamente. Para cada teste mecânico, definimos o conjunto ${ }^{7}$ de dados:

$$
\mathcal{T}_{j}=\left\{\left(\epsilon_{i}, \sigma_{i}\right)\right\}_{i=1}^{a_{j}}, j=1,2, \ldots, k
$$

Determinamos o grau $n_{j}$ do polinômio interpolador do teste mecânico $\mathcal{T}_{j}$, utilizando o teste do $\mathrm{R}$ quadrado [1], denotado por $r^{2}$, quando obtemos $r^{2} \approx 0.9999$ :

$$
r^{2}=1-\frac{\sum_{i=1}^{a_{j}}\left(\sigma_{i}-\sigma_{i}^{*}\right)^{2}}{\sum_{i=1}^{a_{j}}\left(\sigma_{i}-\bar{\sigma}_{i}\right)^{2}},
$$

onde $\sigma_{i}$ são os valores de tensão dos dados, $\sigma_{i}^{*}$ representam os valores de tensão calculados a partir do polinômio interpolador para cada valor de deformação $\epsilon_{i}$ e $\bar{\sigma}_{i}$ é a média dos valores de tensão, com $i=1,2, \ldots, a_{j}$.

Reduzimos então o número de dados de $a_{j}$ para $m_{j}$ no teste $\mathcal{T}_{j}$, de modo que $n_{j}+1=$ $m_{j}$, e interpolamos a curva condicionando o grau do polinômio ao número de dados, obtendo os coeficientes do polinômio através da equação (7). Denotamos por $\psi_{j}$ o polinômio interpolador do teste $\mathcal{T}_{j}$.

\subsection{Ajuste de curvas estimado para compósitos não testados mecanica- mente}

Estimamos valores de propriedades mecânicas em diferentes concentrações não testadas mecanicamente a partir do ajuste de curvas estimado, que será descrito a seguir.

Sejam $\mathcal{T}_{j}$, com $j=1,2, \ldots, k, k$ testes mecânicos nas porcentagens $\gamma_{j}$ de reforço e sejam $\psi_{j}$ seus respectivos polinômios interpoladores. Definimos $n=\max _{j}\left\{n_{j}\right\}_{j=1}^{k}$ e determinamos o conjunto:

$$
\mathcal{S}_{j}=\left\{\left(\epsilon_{i}^{j}, \psi_{j}\left(\epsilon_{i}^{j}\right)\right)\right\}_{i=1}^{n},
$$

para $j=1,2, \ldots, k$, onde $\epsilon_{i}^{j}=i\left(\frac{\text { Deformação máxima (\%) para o teste } \mathrm{T}_{j}}{n}\right)$ e $\psi_{j}\left(\epsilon_{i}^{j}\right)$ é a tensão.

Seja $\mathcal{T}_{\gamma}^{*}$ o teste mecânico de determinado material na porcentagem de concentração $\gamma$ de reforço, para o qual iremos estimar um polinômio interpolador de grau $n$, denotado por $\psi_{\gamma}^{*}$, a partir dos dados do conjunto $\mathcal{S}_{\gamma}^{*}$ :

$$
\mathcal{S}_{\gamma}^{*}=\{(0,0)\} \cup\left\{\left(\epsilon_{i}^{\gamma}, \psi_{\gamma}^{*}\left(\epsilon_{i}^{\gamma}\right)\right)\right\}_{i=1}^{n} .
$$

Definimos a matriz de Vandermonde $\mathcal{A}=\left[a_{j r}\right]_{k \times k}$, onde $a_{j r}=\left(\gamma_{j}\right)^{(r-1)}$ e denotamos os vetores $\vec{v}=\left[c_{0}, c_{1}, \ldots, c_{k-1}\right], \vec{\epsilon}^{i}=\left[\epsilon_{i}^{1}, \epsilon_{i}^{2}, \ldots, \epsilon_{i}^{k}\right]$ e $\vec{\sigma}^{i}=\left[\psi_{1}\left(\epsilon_{i}^{1}\right), \psi_{2}\left(\epsilon_{i}^{2}\right), \ldots, \psi_{k}\left(\epsilon_{i}^{k}\right)\right]$.

\footnotetext{
${ }^{7} \mathrm{O}$ número natural $a_{j}$ no conjunto $\left\{\left(\epsilon_{i}, \sigma_{i}\right)\right\}_{i=1}^{a_{j}}$ denota o número total de dados de deformação $\times$ tensão.
} 
Além disso, definimos o polinômio:

$$
q_{k-1}(\gamma)=\sum_{j=0}^{k-1} c_{j} \gamma^{j} .
$$

Assim, para $i \in\{1,2, \ldots, n\}$, o cálculo $\vec{v}=\mathcal{A}^{-1} \vec{\epsilon}^{i}$ fornece os coeficientes do polinômio $q_{k-1}(\gamma)$. Dado $\gamma$, temos que $q_{k-1}(\gamma)=\epsilon_{i}^{\gamma}$. De maneira similar, se definirmos e calcular$\operatorname{mos} \vec{v}=\mathcal{A}^{-1} \vec{\sigma}^{i}$, então para um dado $\gamma$, temos que $q_{k-1}(\gamma)=\psi_{\gamma}^{*}\left(\epsilon_{i}^{\gamma}\right)$.

Através do conjunto na equação (11), obtemos o polinômio interpolador de grau $n$ para o teste $\mathcal{T}_{\gamma}^{*}$.

\section{Resultados}

O método descrito na Seção 3 foi aplicado em dados de deformação $\times$ tensão de compósitos de polipropileno com fibras de vidro ${ }^{8}$, onde $\epsilon$ está em porcentagem de deformação e a tensão $\sigma$ em megapascal $\left(\mathrm{MPa}=\mathrm{N} / \mathrm{mm}^{2}\right)$. Neste trabalho, desconsideramos o parâmetro temporal $t$.

Três testes em diferentes porcentagens de concentração foram realizados. Definimos os conjuntos de dados obtidos em cada testes mecânico como:

$$
\begin{aligned}
& \mathcal{T}_{1}=\left\{\left(\epsilon_{i}, \sigma_{i}\right)\right\}_{i=1}^{799} \quad \text { (Dados do PP). } \\
& \mathcal{T}_{2}=\left\{\left(\epsilon_{i}, \sigma_{i}\right)\right\}_{i=1}^{294} \quad\left(\text { Dados para } \mathrm{FRP}_{20 \%}\right) . \\
& \mathcal{T}_{3}=\left\{\left(\epsilon_{i}, \sigma_{i}\right)\right\}_{i=1}^{315} \quad\left(\text { Dados para } \mathrm{FRP}_{30 \%}\right) .
\end{aligned}
$$

Nas equações (13)-(15), temos, respectivamente, as seguintes notações: PP denota o compósito de polipropileno puro e $\mathrm{FRP}_{\gamma_{j}}$ denota o compósito de polipropileno com a porcentagem $\gamma_{j}(j=1,2)$ de concentração de fibra de vidro, sendo $\gamma_{1}=20 \%$ e $\gamma_{2}=30 \%$.

Representamos na Tabela 1, os resultados do comportamento mecânico desses compósitos, obtidos a partir das funções $\psi_{j}$.

\begin{tabular}{|c|c|c|c|c|}
\hline Material & $\begin{array}{c}\text { Deformação } \\
\text { Máxima } \\
\%\end{array}$ & $\begin{array}{c}\text { Tensão } \\
\text { Máxima } \\
\mathrm{MPa}\left(10^{6} \mathrm{~N} / \mathrm{m}^{2}\right)\end{array}$ & $\begin{array}{c}\text { Módulo de } \\
\text { Resiliência } \\
\mathrm{J} / \mathrm{m}^{3}\end{array}$ & $\begin{array}{c}\text { Módulo de } \\
\text { Young } \\
\mathrm{GPa}\left(10^{9} \mathrm{~N} / \mathrm{m}^{2}\right)\end{array}$ \\
\hline $\mathrm{PP}$ & 5.77 & 38.57 & 157.56 & 1.505 \\
\hline $\mathrm{FRP}_{15 \%}$ & 2.53 & 49.83 & 68.48 & 2.871 \\
\hline $\mathrm{FRP}_{20 \%}$ & 2.11 & 56.55 & 64.08 & 3.324 \\
\hline $\mathrm{FRP}_{25 \%}$ & 2.02 & 64.76 & 71.51 & 3.776 \\
\hline $\mathrm{FRP}_{30 \%}$ & 2.26 & 74.45 & 95.30 & 4.227 \\
\hline $\mathrm{FRP}_{35 \%}$ & 2.84 & 85.63 & 143.31 & 4.677 \\
\hline
\end{tabular}

Tabela 1: Propriedades mecânicas para os testes e estimativas.

\footnotetext{
${ }^{8}$ Os testes de deformação e tensão foram realizados de acordo com as normas ASTM D3039 de materiais compósitos e ASTM D638 de plásticos, conforme especificados pela American Society for Testing and Materials (ASTM).
} 
Na Fig. 2, temos a representação gráfica da metodologia descrita para os compósitos de polipropileno com fibras de vidro.

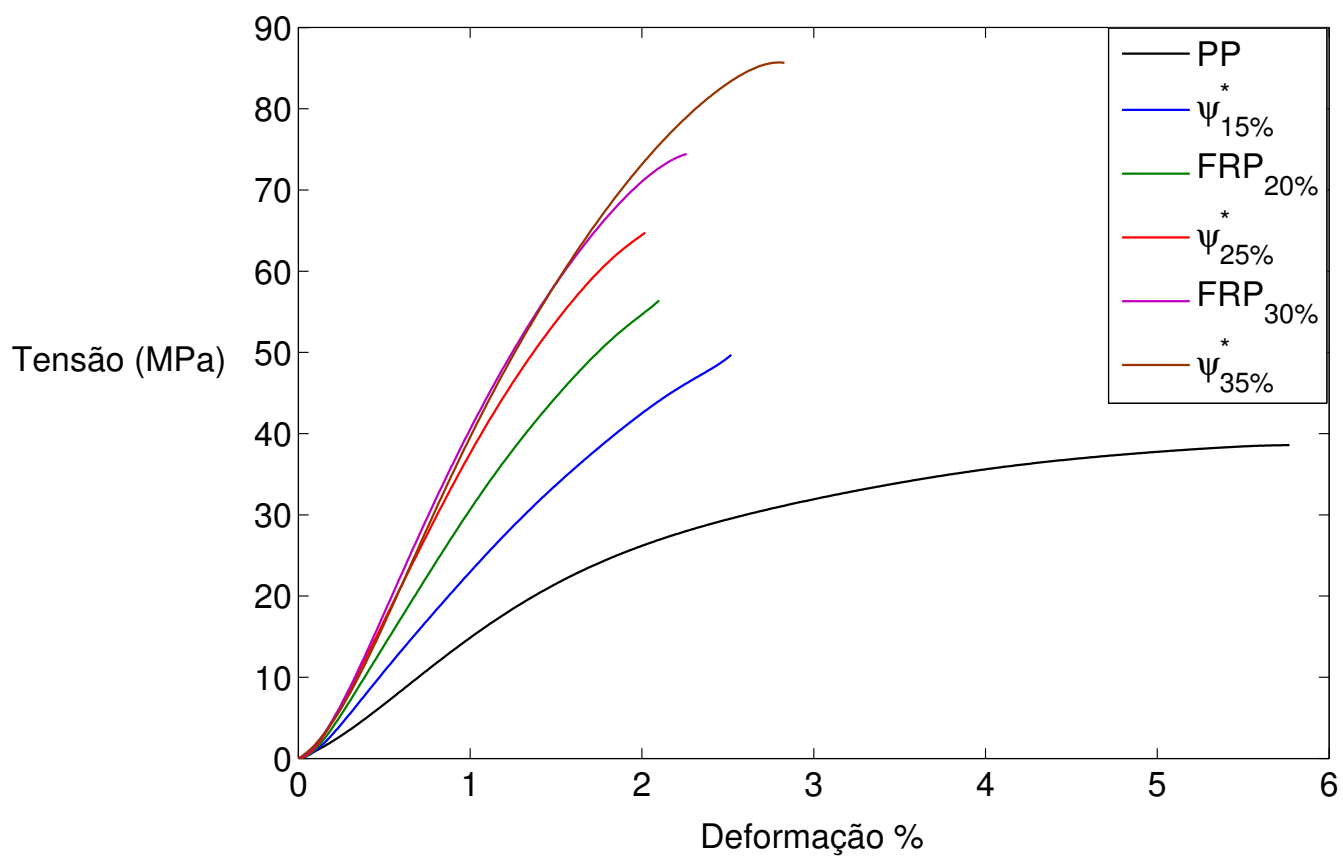

Figura 2: Testes mecânicos $\mathrm{FRP}_{\gamma_{j}}$ e estimativas $\psi_{\gamma}^{*}$.

Para os testes realizados e analisados neste trabalho, o ponto UTS coincidiu com o ponto de quebra dos compósitos por se tratarem de compósitos de polipropileno com fibras de vidro.

\section{Conclusões}

Um ajuste não linear através de curvas polinomiais dos dados de testes mecânicos de tração e flexão de corpos de prova nos possibilitou determinar: a tensão máxima de tração, o limite de ruptura e os módulos de resiliência e Young, que por sua vez, proporcionaram ainda dados para estimar a função polinomial para compósitos não testados mecanicamente. Usando essa estimativa matemática, reduzimos a necessidade de realizar mais testes mecânicos em outras concentrações.

\section{Agradecimentos}

Os autores agradecem a FAPESP por financiar esta pesquisa. 


\section{Referências}

[1] J. L. Devore. Probability and statistics for engineering and the sciences. MA: Cengage Learning, Boston, $8^{\text {a }}$ ed., 2011.

[2] J. González-Benito and D. Olmos. Efficient dispersion of nanoparticles in thermoplastic polymers. Society of Plastics Engineers. Plastics Research Online, 2010.

[3] F. Guild, Quasielastic mechanical properties. Polymer blends. New York: John Wiley \& Sons, volume 2, 2000.

[4] F. Mainardi. Fractional calculus and waves in linear viscoelasticity. Imperial College Press. London, 2000.

[5] V. V. Mitin, D. I. Sementsov and N. Z. Vagidov. Quantum mechanis for nanostructures. Cambridge University Press. New York, 2010.

[6] W. M. Silva, M. V. A. Soeiro and E. C. Grigoletto. Mechanical properties evaluation of composite depending on wight concentrations of natural fiber. 22 ${ }^{\circ}$ CBECiMat Congresso Brasileiro de Engenharia e Ciência dos Materiais, volume 1, pages 42164227, 2016.

[7] M. Supová, G. S. Martynková and K. Barabaszová. Effect of nanofillers dispersion in polymer matrices: a review. Science of Advanced Materials, volume 3, pages 1-25, 2011.

[8] J. A. Tenreiro Machado, V. Kiryakova and F. Mainardi, A molecular dynamics simulation study of nanoparticle interactions in a model polymer-nanoparticle composite. Composites Science and Technology, volume 63, pages 1599-1605, 2003. 